\title{
Carrier dynamics in dilute II-VI oxide highly mismatched alloys
}

\author{
Yan-Cheng Lin*a, Wu-Ching Chou ${ }^{\mathrm{a}}$, Jen-Inn Chyi ${ }^{\mathrm{b}}$, Tooru Tanaka ${ }^{\mathrm{c}}$ \\ ${ }^{a}$ Department of Electrophysics, National Chiao Tung University, Hsinchu 30010, Taiwan \\ ${ }^{b}$ Department of Electrical Engineering, National Central University, Jhongli 32001,Taiwan \\ ${ }^{c}$ Department of Electrical and Electronic Engineering, Saga University, Saga 840-8502, Japan
}

\begin{abstract}
This study explores comprehensively the carrier dynamics in $\mathrm{ZnSeO}$ and $\mathrm{ZnTeO}$ using photoluminescence (PL) and time-resolved PL spectroscopy. As the O concentration increases, the PL emissions shift toward lower energies. Additionally, the PL lifetime increases with increasing $\mathrm{O}$ contents and the decay curves exhibit complex behavior. In the case of $\mathrm{ZnSeO}$, the mechanism of carrier recombination undergoes a complicated change from trapped to free excitons with the increase in temperature. The incorporation of $\mathrm{O}$ in $\mathrm{ZnTe}$ generates a wide distribution of electron localization below the energy of the $E$. conduction subband, and these cause broad PL emission and serve as another intermediate

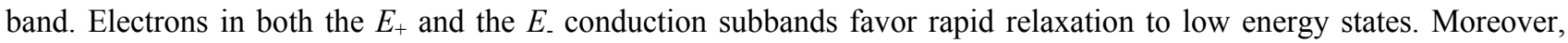
temperature-independent long carrier lifetimes ( $>130.0 \mathrm{~ns}$ ) that are induced by localized electrons increase with $\mathrm{O}$ concentration.
\end{abstract}

Keywords: II-VI oxides, highly mismatched alloys, time-resolved photoluminescence, carrier dynamics, $\mathrm{ZnSeO}, \mathrm{ZnTeO}$, intermediate band

\section{INTRODUCTION}

An urgent and global need for renewable energy sources has engendered extraordinary research interest in potential photovoltaic materials, which aims to the gradual phasing out of conventional and nuclear power. Oxides are promising owing to their natural availability, environmental stability, and ecologically-friendly characteristics, yet traditionally limited for applications in photovoltaics by a wide band gap. Because of its large electronegativity, oxygen forms chemical bonds with almost all elements to give the corresponding oxides. Thus, band gap engineering of these highly attractive oxide materials could make them accessible for photoelectron and solar cell applications.

Dilute II-VI oxide semiconductors whose constituent anions are partially substituted by isovalent oxygen atoms of distinctly different electronegativity and size, are commonly referred to as highly mismatched alloys (HMAs).

*bryanlin@mail.nctu.edu.tw; phone +886-3-571-2121-56171; fax +886-3-572-5230

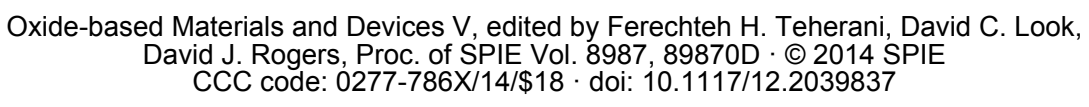


$\mathrm{ZnSeO}$ and $\mathrm{ZnTeO}$ are the most widely studied examples of such HMAs, exhibiting multiple band gaps and giant band gap bowing. ${ }^{1-8}$ The unusual energy band structure of these HMAs forms according to the band anticrossing between the defect states of the substitutional minority $\mathrm{O}$ anions and the extended states of the host semiconductor matrix. ${ }^{4,9}$ Since the electronegativity of the incorporated $\mathrm{O}$ atom is greater than those of the host $\mathrm{Se}$ and $\mathrm{Te}$ atoms, isovalent defect states form near the conduction band edge (CBE). Accordingly, the interactions that occur in $\mathrm{ZnSeO}$ and $\mathrm{ZnTeO}$ are thought to be the $\mathrm{CB}$ band anticrossing (CBAC). As a result the $\mathrm{CB}$ splits into two subbands ( $E_{+}$and $\left.E_{-}\right)$with distinctly nonparabolic dispersion relations. ${ }^{4,9} \mathrm{In} \mathrm{ZnSeO}$, the $\mathrm{O}$ defect state is located within the $\mathrm{CB}$ of $\mathrm{ZnSe}$, so a wide lower $E_{\text {. }}$ band is formed through the anticrossing effect. However, a narrow lower band can be formed only if the defect state lies well below the $\mathrm{CBE}$, and this situation occurs in $\mathrm{ZnTeO} .{ }^{4}$ Additionally, the strength of the anticrossing depends on the mismatch of electronegativities, which is more pronounced in $\mathrm{ZnTeO}$ than in $\mathrm{ZnSeO}$ because the mismatch between $\mathrm{O}$ and $\mathrm{Te}(\Delta X=1.4)$ exceeds that between $\mathrm{O}$ and $\mathrm{Se}(\Delta X=1.1)$. Therefore, in $\mathrm{ZnTeO}$, the naturally formed narrow $E_{-}$band is highly separated from the higher-lying $E_{+}$subband and acts as an intermediate band (IB), which provides additional optical transitions.

Although much research effort has focused on $\mathrm{ZnSeO}$ and $\mathrm{ZnTeO}$, the unique decay dynamics resulted from $\mathrm{O}$ in these materials remains unexplored. Recently, we demonstrated that an increase in the number of isoelectronic Te localized traps complicates the carrier relaxation paths in ZnSeTe at low temperature. ${ }^{10,11}$ In this work, we report results on the temperature-dependent photoluminescence (PL) and time-resolved PL (TRPL) of $\mathrm{ZnSeO}(\mathrm{O}=2.7$ and $5.3 \%$ ) and $\mathrm{ZnTeO}$ $(\mathrm{O}=0.43,0.77$, and $1.09 \%)$. Complex recombination paths are clarified using the relaxation model based on various decay channels. The aim of this study is to investigate thoroughly the influence of both $\mathrm{O}$ and temperature on the recombination dynamics in these semiconductors, which are important for further optoelectronic applications.

\section{EXPERIMENT}

$\mathrm{ZnSeO}(\mathrm{O}=2.7$ and $5.3 \%)$ films were grown by RF-plasma assisted molecular beam exitaxy on (001) GaAs substrates following the deposition of a $100 \mathrm{~nm}$-thick ZnSe buffer layer. The thicknesses of all films were fixed at around $0.5 \mu \mathrm{m}$. The $\mathrm{O}$ content was determined by $\mathrm{x}$-ray diffraction (XRD) and energy-dispersive $\mathrm{x}$-ray analyses. PL and TRPL were excited using a $200 \mathrm{ps}$ pulsed laser diode $(405 \mathrm{~nm} / 10 \mathrm{MHz})$ with an excitation power of about $1 \mathrm{~mW}$. An $\mathrm{He}-\mathrm{Cd}(325 \mathrm{~nm})$ laser was used to make excitation power-dependent PL measurements. Signals were dispersed using a Horiba iHR550 spectrometer with a $1800 \mathrm{gr} / \mathrm{mm}$ grating, and detected using photomultiplier tubes.

$\mathrm{ZnTeO}(\mathrm{O}=0.43,0.77$, and $1.09 \%)$ films were grown by radical source molecular beam epitaxy on $\mathrm{ZnTe}(001)$ substrates, following a procedure that has been published previously. ${ }^{7}$ The thickness of all films was $0.6 \mu \mathrm{m}$. Their O content was determined by high-resolution XRD analysis, as described elsewhere. ${ }^{7}$ PL and TRPL were excited using 200 ps pulsed laser diodes ( 377 or $638 \mathrm{~nm} / 2.5 \mathrm{MHz} / 1 \mathrm{~mW}$ ). A $377 \mathrm{~nm}$ continuous wave laser was used to make excitation 
power-dependent PL measurements. The PL and TRPL signals were dispersed using a Horiba iHR320 spectrometer with a $1200 \mathrm{gr} / \mathrm{mm}$ grating, and detected using an $\mathrm{LN}_{2}$-cooled InGaAs photodiode and an Si photon-counting avalanche photodiode. The decay traces were recorded by time-correlated single photon counting (Time-Harp, PicoQuant).

\section{RESULTS AND DISCUSSION}

\subsection{ZnSeO}

Figure 1 (a) shows the PL spectra of $\mathrm{ZnSeO}$ at $10 \mathrm{~K}$. Clearly, a sharp near-band-edge emission of $\mathrm{ZnSe}$ is observed at around $2.80 \mathrm{eV}$. When $\mathrm{O}$ is substituted into $\mathrm{ZnSe}$, the PL emissions shift toward lower energies, which shift is accompanied by asymmetric linewidth broadening with increasing $\mathrm{O}$ content. The large energy redshift matches the O-induced band-gap bowing, caused by an anticrossing between the $\mathrm{O}$ states and the ZnSe CB states. The PL linewidth broadening is attributable to alloy fluctuations or O clustering. Excitation power-dependent PL measurements of $\mathrm{ZnSeO}$ $(\mathrm{O}=2.7 \%)$ at $10 \mathrm{~K}$ show a redshift of the PL peak as the laser power decreases. The PL peaks shift by about 8 meV as the laser power is decreased by three orders of magnitude. A ZnSe reference sample exhibited no change in peak position over the same decrease in power. This feature is typical of carrier localization and filling effect.
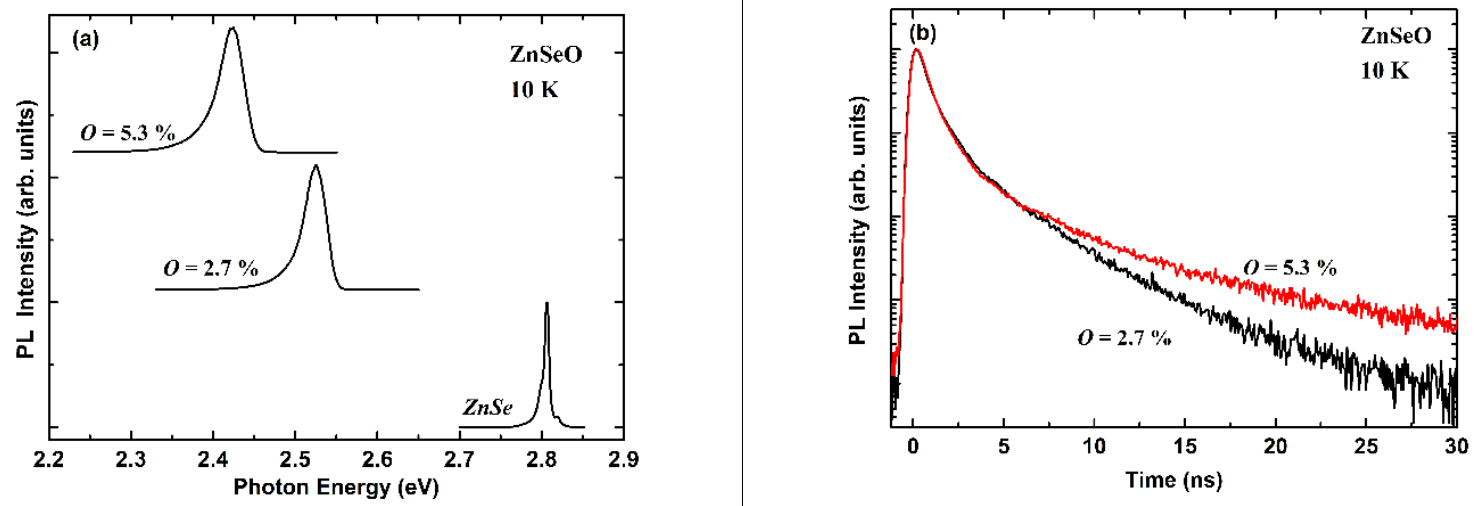

Figure 1. (a) PL and (b) TRPL spectra of $\mathrm{ZnSe}$ and $\mathrm{ZnSeO}$ at $10 \mathrm{~K}$. Figure modified from ${ }^{[3]}$

Figure 1(b) shows the TRPL spectra of $\mathrm{ZnSeO}$ monitored at the PL peaks and $10 \mathrm{~K}$. The PL decay profiles reveal nonsingle-exponential decay and can be decomposed into a fast (initial) and a slow (tail) component. The fast component is a mono-exponential decay, while the slow component is stretched-exponential decay. Obviously, the PL lifetime of the slow component significantly increases with the $\mathrm{O}$ concentration. The decay curves are fitted using the following equation,

$$
I(t)=I_{1} e^{-\left(t / \tau_{1}\right)}+I_{2} e^{-\left(t / \tau_{2}\right)^{\beta}}
$$

where $\beta$ is the stretching exponent. This stretching exponent measures the relaxation rates involved in the PL decay 
process, a smaller $\beta$ indicates a broader rate distribution. The fitted $\tau_{1}$ is around $850 \mathrm{ps}$ for both samples; $\tau_{2}(\beta)$ is around $2.8 \mathrm{~ns}(0.96)$ and $4.1 \mathrm{~ns}(0.93)$ for $\mathrm{O}=2.7$ and $5.3 \%$, respectively. Increasing the $\mathrm{O}$ concentration increases $\tau_{2}$ and reduces $\beta$, implying that the slow decay originates in localized excitons (LE) due to an increasing number of trap states and decay paths. This suggestion is consistent with the hopping-transport model, in which the concentrations of the transport and trapping sites determine the $\beta .^{12}$

To clarify further the dependence of the carrier dynamics in $\mathrm{ZnSeO}$ on temperature, temperature-dependent PL of $\mathrm{ZnSeO}$ $(\mathrm{O}=5.3 \%)$ is studied. As shown in Fig. 2(a), the PL peaks exhibit an S-shaped energy shift as the temperature is increased, which feature is typical of carrier localization. At low temperatures, a single broad band from LE dominates the PL emissions. As the temperature increases, emission from LE is rapidly thermally quenched, disappearing beyond $100 \mathrm{~K}$. When the temperature is increased above $70 \mathrm{~K}$, another peak appears as a higher energy shoulder of the dominant peak; it becomes more pronounced as the temperature is increased further. This emission exhibits a normal Gaussian shape $(\mathrm{T}>120 \mathrm{~K})$, while that from LE at $10 \mathrm{~K}$ is asymmetric, with a low-energy tail and a sharp high-energy cutoff. Moreover, this emission exhibits no obvious energy shift as the excitation power is varied by four orders of magnitude. Based on these observations, the emission at the high energy shoulder is attributed to FE, which are thermally detrapped from the isoelectronic $\mathrm{O}$ trap states.
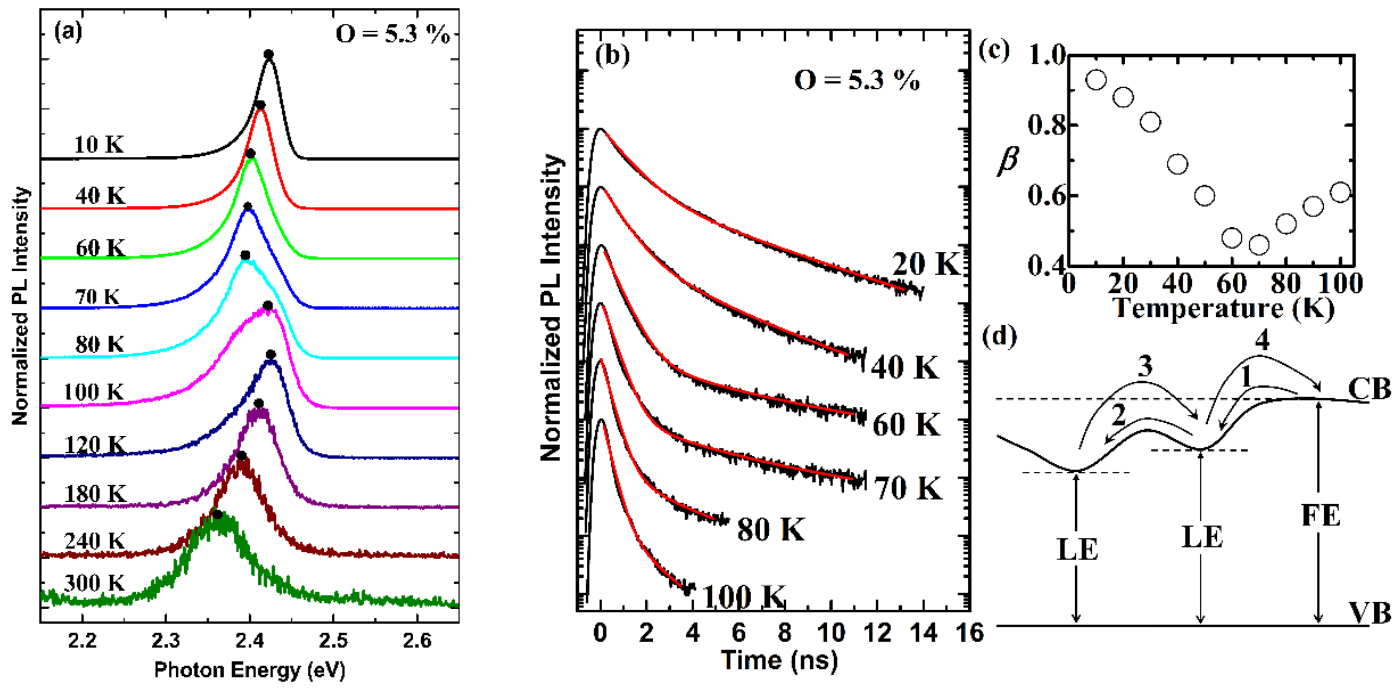

Figure 2. Temperature-dependent (a) PL and (b) TRPL spectra of $\mathrm{ZnSeO}(\mathrm{O}=5.3 \%)$. (c) Stretching exponent $\beta$ of $\mathrm{ZnSeO}$ $(\mathrm{O}=5.3 \%)$. (d) Schematic diagram indicates complex relaxation channels. For simplicity only two trap states are considered. Figure modified from ${ }^{[3]}$

Figure 2(b) shows the temperature-dependent TRPL spectra of $\mathrm{ZnSeO}(\mathrm{O}=5.3 \%)$. Increasing the temperature significantly reduces the lifetime of the slow component. As the temperature is increased above $100 \mathrm{~K}$, the slow decay component is eliminated, and the fast decay dominates the entire decay profile. Furthermore, the bending of the 
logarithmic decay curve, reflecting a reduction in $\beta$, initially becomes more pronounced as the temperature increases to $70 \mathrm{~K}$ and then slowly diminishes, such that $\beta$ begins to increase. To measure $\beta$ quantitatively, the decay curves are fitted using Eq. (1). Figure 2(c) plots $\beta$ against temperature. As the temperature increases, $\beta$ initially decreases to a minimum at $70 \mathrm{~K}$ and then monotonically increases with a further increase in temperature. This phenomenon can be explained by the configuration coordinate diagram shown in Fig. 2(d). At $10 \mathrm{~K}$, electrons that are generated initially in the free state hop among proximal transport and trapping sites, and recombine with $\mathrm{O}$ traps (channel 1). As the temperature increases, these less mobile electrons gain additional energy that enables them to hop to deeper trap states (channel 2), causing a rapid redshift in the LE peak and reducing $\beta$. Simultaneously, as the temperature approaches $70 \mathrm{~K}$, some of the trapped electrons are thermally activated to repopulate the higher energy states (channel 3), leading to significant PL linewidth broadening at the high energy shoulder. At still higher temperatures, an increasing number of electrons gain sufficient energy to delocalize into the free state (channel 4) and recombine, explaining the increase in $\beta$ and the blueshift of the PL peaks. Above $100 \mathrm{~K}$, the slow decay component disappears because FE dominates the recombination, resulting in a monotonic PL energy redshift with the temperature.

\subsection{ZnTeO}

Figure 3(a) shows the PL spectra of $\mathrm{ZnTeO}(\mathrm{O}=0.43,0.77$, and $1.09 \%)$ at $10 \mathrm{~K}$. Notably, only broad multi-peak emissions below $2.00 \mathrm{eV}$ can be observed. Such broad emissions cannot be simply attributed to the direct $E_{\text {--valance }}$ band (VB) transition. Additionally, as the O content increases, the PL emissions shift rapidly downward in energy. The large PL energy redshift and the lack of ZnTe near-band-edge (NBE) emission upon the incorporation of $\mathrm{O}$ are strong evidence of the O-induced CBAC effect in $\mathrm{ZnTeO}$. Accordingly, the absence of the $E_{+}-\mathrm{VB}$ transition in $\mathrm{ZnTeO}$ is attributable to the fast electron relaxation from the $E_{+}$to the $E_{\text {- }}$ or lower energy states and will be discussed later. Figure 3(b) shows the excitation power-dependent PL spectra of $\mathrm{ZnTeO}(\mathrm{O}=1.09 \%)$ at $10 \mathrm{~K}$. The PL peaks are redshifted by $100 \mathrm{meV}$ and exhibited asymmetric bandwidth broadening as the laser power is reduced by two orders of magnitude. The huge PL peak energy redshift with decreasing power reveals that the density of states exhibits an exponential tail, which arises from $\mathrm{O}$ clustering or alloy fluctuations, indicating possible carrier localization. The electronic states that

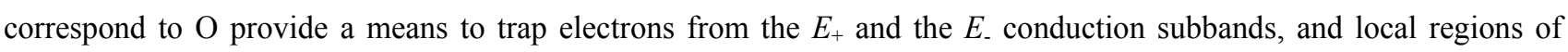
higher $\mathrm{O}$ concentration are associated with trapping states of lower energy.

Figure 3(c) shows the TRPL spectra at $10 \mathrm{~K}$. Clearly, the PL lifetimes increase with the O content and are in the range of several tens to a few hundreds of nanoseconds. The PL decay traces exhibit unusual (long decay lifetimes) and complex (curved slow profile) carrier decay dynamics. To analyze the TRPL data, a four-level rate equation model, schematically depicted in Fig. 3(d), was first adopted. However, the standard analysis procedure fails to correlate well with the curved slow decay component, implying a wide and continuous distribution of $\mathrm{O}$ traps. Therefore, to obtain a more quantitative measure of the decay behavior, the decay curves are all fitted using equation (1). Table I summarizes the decay 
coefficients that are derived from the fits to experimental data. Increasing the $\mathrm{O}$ concentration markedly increases $\tau_{2}$ and reduces $\beta$. These phenomena are signatures of II-VI HMAs due to the increasing number of additional decay paths for electrons, implying that the slow decay originates in LE. Moreover, increasing the $O$ content substantially reduces/increases the magnitude of the fast/slow decay component $\left(I_{1} / I_{2}\right)$. These facts reveal that the entire PL decay profile gradually becomes a simple stretched exponential decay,

$$
I(t)=I_{0} e^{-(t / \tau)^{\beta}},
$$

where the decay curve on a double logarithmic scale is a straight line, as is the case for $\mathrm{ZnTeO}(\mathrm{O}=1.09 \%)$, displayed in the inset in Fig. 3(c).
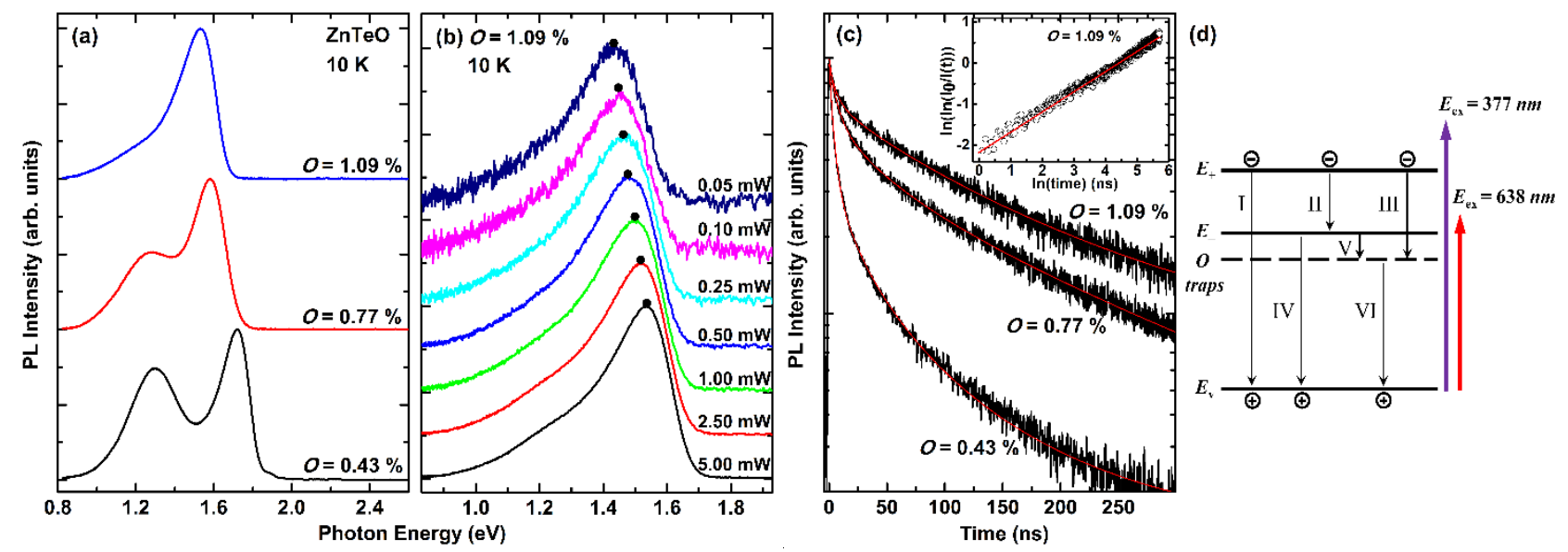

Figure 3. (a) PL (under $377 \mathrm{~nm}$ excitation), (b) excitation power-dependent PL, and (c) TRPL spectra of ZnTeO. Inset in (c) shows TRPL spectrum of $\mathrm{ZnTeO}(\mathrm{O}=1.09 \%)$ on a double logarithmic scale. (d) Schematic energy band diagram and associated optical transitions of $\mathrm{ZnTeO}$. For simplicity, only one $\mathrm{O}$ trapping state is considered. Figure modified from ${ }^{[13]}$

Table I. Fitting parameters of TRPL spectra in Fig. 3(c).

\begin{tabular}{cccccc}
\hline \hline O Content $(\%)$ & $I_{1}(\%)$ & $\tau_{1}(\mathrm{~ns})$ & $I_{2}(\%)$ & $\tau_{2}(\mathrm{~ns})$ & $\beta$ \\
\hline 0.43 & 60 & 3.6 & 40 & 29.0 & 0.70 \\
0.77 & 20 & 6.0 & 80 & 106.5 & 0.62 \\
1.09 & 5 & 6.3 & 95 & 134.8 & 0.52 \\
\hline \hline
\end{tabular}

Figure 3(d) shows the energy band diagram and the associated optical transitions of $\mathrm{ZnTeO}$. Under $377 \mathrm{~nm}$ excitation, the initial fast decay of $\mathrm{ZnTeO}$ can be ascribed to the following effects. (i) The radiative recombination of electrons

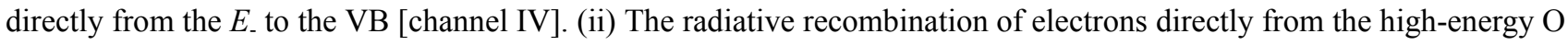


traps (electrons that do not hop toward low-energy traps) to the VB [channels VI]. The nonradiative recombination is negligible since the PL is dominated by the radiative recombination at low temperature. The direct $E_{-}-\mathrm{VB}$ transition could occur only in $\mathrm{ZnTeO}(\mathrm{O}=0.43 \%)$ because its PL emission at high energy shoulder is close to the transition edge.

To verify this claim, Fig. 4(a) shows the TRPL spectra of $\mathrm{ZnTeO}(\mathrm{O}=0.43 \%)$ at various monitored energies. Obviously, the decay time from the slow component sharply falls as the monitored energy increases, while that from the fast component is insensitive to energy. The values of $\tau_{1}, \tau_{2}$, and $\beta$ are $1.7 / 5.8 \mathrm{~ns}, 10.4 / 83.5 \mathrm{~ns}$, and $0.72 / 0.52$, respectively, at $1.83 / 1.28 \mathrm{eV}$. This behavior implies that the electrons transfer from shallow to deep $\mathrm{O}$ traps. Moreover, monoexponential decay dominates the overall PL decay profile at high energy, becoming even more pronounced upon the recording of the high-energy emissions. The above results confirm the contribution of the $E_{-}-\mathrm{VB}$ transition in $\mathrm{ZnTeO}(\mathrm{O}=0.43 \%)$, which is responsible for the huge initial rapid decay, as shown in Fig. 3(c). Hence, the recombination of electrons directly from the higher lying $\mathrm{O}$ traps to the $\mathrm{VB}$ is the main cause of the initial fast decay in $\mathrm{ZnTeO}(\mathrm{O}=0.77$ and $1.09 \%)$.

The TRPL spectra of $\mathrm{ZnTeO}(\mathrm{O}=0.77 \%)$ at $10 \mathrm{~K}$ under 377 and $638 \mathrm{~nm}$ excitation, shown in Fig. 4(b), further confirm the aforementioned fast carrier decay. Clearly, the spectrum obtained under $638 \mathrm{~nm}$ excitation exhibits faster initial decay and a larger initial decay component than that obtained using the $377 \mathrm{~nm}$ laser. Restated, the initial fast decay is greatly suppressed under $377 \mathrm{~nm}$ excitation. Similar results were observed in $\mathrm{ZnTeO}(\mathrm{O}=0.43$ and 1.09 \%). These phenomena are attributable to the feeding of photo-excited electrons from the $E_{+}$to low-energy states [channels II and III in Fig. 3(d)], which increases the carrier delay times of channels IV and VI during the first few nanoseconds following

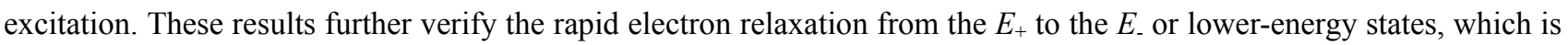
responsible for the absence of the PL emission associated with direct $E_{+}-\mathrm{VB}$ transition [channel I in Fig. 3(d)].
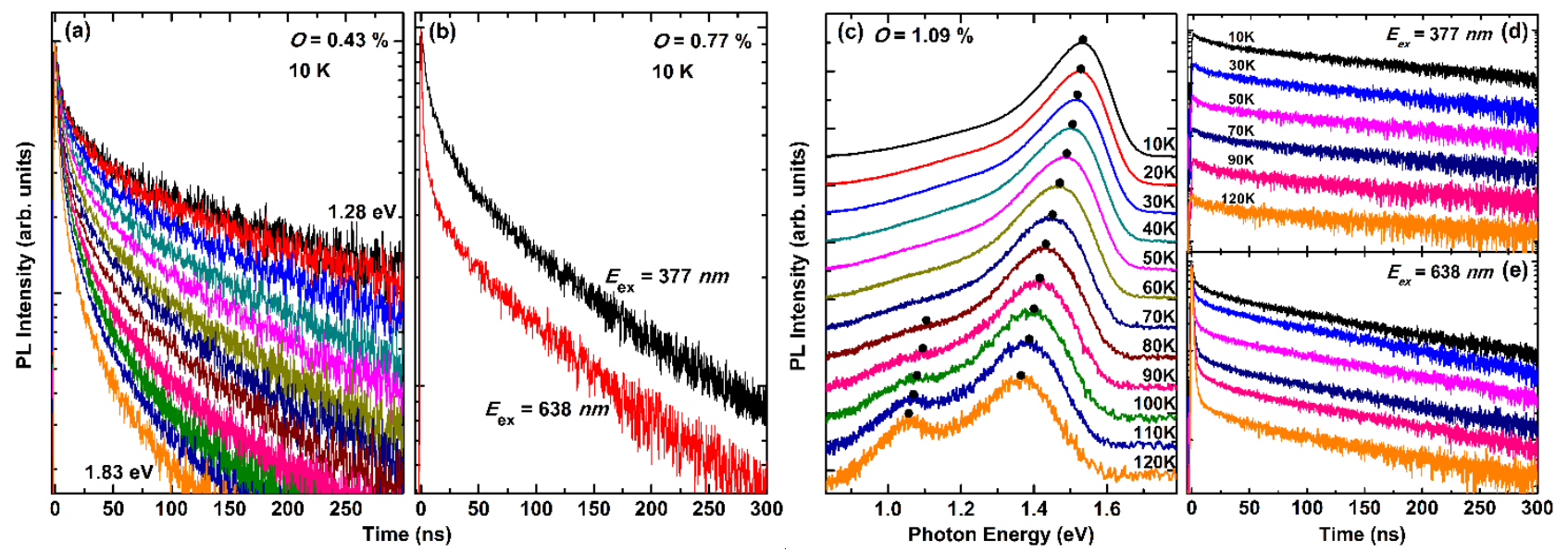

Figure 4. TRPL spectra of (a) $\mathrm{ZnTeO}(\mathrm{O}=0.43 \%)$ for monitored energies from 1.83 to $1.28 \mathrm{eV}$ under $377 \mathrm{~nm}$ excitation and (b) $\mathrm{ZnTeO}(\mathrm{O}=0.77 \%)$ monitored at PL peak under 377 and $638 \mathrm{~nm}$ excitation. (c) Temperature-dependent PL spectra 
of $\mathrm{ZnTeO}(\mathrm{O}=1.09 \%)$ under $377 \mathrm{~nm}$ excitation and corresponding TRPL spectra obtained at PL peaks under (d) $377 \mathrm{~nm}$

and (e) $638 \mathrm{~nm}$ excitation. Figure modified from ${ }^{[13]}$

Figure 4(c) shows the temperature-dependent PL spectra of $\mathrm{ZnTeO}(\mathrm{O}=1.09 \%)$ with peak positions indicated. As the temperature increases, the PL peaks shift monotonically toward lower energies; these shifts are accompanied by asymmetric bandwidth broadening. The PL peaks are redshifted by $\sim 170 \mathrm{meV}(10-120 \mathrm{~K})$, which is larger than the shift of ZnTe NBE emission ( $120 \mathrm{meV} ; 15-300 \mathrm{~K})$. This feature is typical of carrier localization in a variable potential that is formed by the distribution of $\mathrm{O}$ traps. Above $70 \mathrm{~K}$, the low-energy peak suddenly appears, which demonstrates that some of the trapped electrons gain extra energy to transfer into deeper O states. Figures 4(d) and 4(e) show the temperature-dependent TRPL spectra of $\mathrm{ZnTeO}(\mathrm{O}=1.09 \%)$ obtained under 377 and $638 \mathrm{~nm}$ excitation, respectively. Surprisingly, as revealed by Fig. 4(d), the decay curves are almost identical throughout the temperature range, except for their emission intensities. However, in Fig. 4(e), under $638 \mathrm{~nm}$ excitation, the initial fast decay, which is very strongly suppressed under $377 \mathrm{~nm}$ excitation, increasingly dominates the entire PL decay as the temperature increases. However, the slow decay remains almost unchanged. These experimental results indicate the emergence of thermally induced nonradiative recombination channels, which trigger the ultra-fast decay at high temperatures.

\section{CONCLUSIONS}

In summary, this study investigated the time-resolved carrier dynamics in dilute II-VI oxide, $\mathrm{ZnSeO}$ and $\mathrm{ZnTeO} \mathrm{HMAs}$ using temperature-dependent PL and TRPL spectroscopy. In $\mathrm{ZnSeO}$, the deduced stretching exponent $\beta$ initially declines to a minimum as the temperature is increased to $70 \mathrm{~K}$, reflecting that the recombination with the trapped electrons is significant at low temperatures. However, at higher temperatures, the free electrons that are thermally delocalized from the traps dominate the recombination. Thus, the recombination with LE gradually disappears, subsequently increasing $\beta$. In $\mathrm{ZnTeO}$, The incorporation of $\mathrm{O}$ generates a wide distribution of electron localization below the energy of the $E$. conduction subband, and these cause broad PL emission and serve as another IB. Decay curves that are induced by O traps exhibit nonsingle yet stretched exponential decay, and the corresponding temperature-independent long radiative lifetime $(\sim 135.0 \mathrm{~ns}$ for $\mathrm{O}=1.09 \%)$ increases with $\mathrm{O}$ concentration. However, electrons in both the $E_{+}$and the $E_{\text {- }}$ bands favor rapid relaxation to low energy states due to the intrinsic p-type behavior of $\mathrm{ZnTeO}$ and the emergence of $\mathrm{O}$ traps.

\section{ACKNOWLEDGEMENT}

This work was supported by the Ministry of Education and the National Science Council under Grant No. NSC 102-2112-M-009-001-MY2. 


\section{REFERENCES}

[1] Shan, W., et al., "Effect of oxygen on the electronic band structure in $\mathrm{ZnO}_{\mathrm{x}} \mathrm{Se}_{1-\mathrm{x}}$ alloys," Appl. Phys. Lett. 83, 299 (2003).

[2] Broesler, R., et al., "Temperature dependence of the band gap of $\mathrm{ZnSe}_{1-\mathrm{x}} \mathrm{O}_{\mathrm{x}}$," Appl. Phys. Lett. 95, 151907 (2009).

[3] Lin, Y. C., et al., "Carrier dynamics in isoelectronic $\mathrm{ZnSe}_{1-\mathrm{x}} \mathrm{O}_{\mathrm{x}}$ semiconductors," Appl. Phys. Lett. 97, 041909 (2010).

[4] Yu, K. M., et al., "Diluted II-VI oxide semiconductors with multiple band gaps," Phys. Rev. Lett. 91, 246403 (2003).

[5] Wang, W., et al., "Intermediate-band photovoltaic solar cell based on ZnTe:O," Appl. Phys. Lett. 95, 011103 (2009).

[6] Tanaka, T., et al., "Demonstration of $\mathrm{ZnTe}_{1-x} \mathrm{O}_{x}$ intermediate band solar cell," Jpn. J. Appl. Phys. 50, 082304 (2011).

[7] Tanaka, T., et al., "Molecular beam epitaxial growth and optical properties of highly mismatched $\mathrm{ZnTe}_{1-\mathrm{x}} \mathrm{O}_{\mathrm{x}}$ alloys," Appl. Phys. Lett. 100, 011905 (2012).

[8] Tanaka, T., et al., "Photocurrent induced by two-photon excitation in ZnTeO intermediate band solar cells," Appl. Phys. Lett. 102, 052111 (2013).

[9] Shan, W., et al., "Band anticrossing in GaInNAs alloys," Phys. Rev. Lett. 82, 1221 (1999).

[10] Lin, Y. C., et al., "Time-resolved photoluminescence of isoelectronic traps in $\mathrm{ZnSe}_{1-\mathrm{x}} \mathrm{Te}_{\mathrm{x}}$ semiconductor alloys," Appl. Phys. Lett. 93, 241909 (2008).

[11]Lin, Y. C., et al., "Temperature-dependent decay dynamics in highly mismatched $\mathrm{ZnSe}_{1-\mathrm{x}} \mathrm{Te}_{\mathrm{x}}$ alloy," Appl. Phys. Lett. 100, 071912 (2012).

[12] Sturman, B., et al., "Origin of stretched exponential relaxation for hopping-transport models," Phys. Rev. Lett. 91, 176602 (2003).

[13]Lin, Y. C., et al., "Recombination dynamics and carrier lifetimes in highly mismatched ZnTeO alloys," Appl. Phys. Lett. 103, 261905 (2013).

Proc. of SPIE Vol. 8987 89870D-9 